\title{
Molecular Prevalence and Identification of Ehrlichia canis and Anaplasma platys from Dogs in Nay Pyi Taw Area, Myanmar
}

\author{
Myint Myint Hmoon, ${ }^{1}$ Lat Lat Htun, ${ }^{1}$ May June Thu, ${ }^{1}$ Hla Myet Chel, ${ }^{1}$ Yu Nandi Thaw, \\ Shwe Yee Win, ${ }^{1}$ Nyein Chan Soe, ${ }^{1}$ Yadanar Khaing, ${ }^{2}$ Su Su Thein, ${ }^{1}$ and Saw Bawm $\mathbb{D}^{2}$ \\ ${ }^{1}$ Department of Pharmacology and Parasitology, University of Veterinary Science, Nay Pyi Taw 15013, Myanmar \\ ${ }^{2}$ Department of International Relations and Information Technology, University of Veterinary Science, \\ Nay Pyi Taw 15013, Myanmar \\ Correspondence should be addressed to Saw Bawm; bestshadow@gmail.com
}

Received 17 May 2020; Revised 23 January 2021; Accepted 30 January 2021; Published 8 February 2021

Academic Editor: Antonio Ortega-Pacheco

Copyright (C) 2021 Myint Myint Hmoon et al. This is an open access article distributed under the Creative Commons Attribution License, which permits unrestricted use, distribution, and reproduction in any medium, provided the original work is properly cited.

\begin{abstract}
Ticks are vectors of different types of viruses, protozoans, and other microorganisms, which include Gram-negative prokaryotes of the genera Rickettsiales, Ehrlichia, Anaplasma, and Borrelia. Canine monocytic ehrlichiosis caused by Ehrlichia canis and canine cyclic thrombocytopenia caused by Anaplasma platys are of veterinary importance worldwide. In Myanmar, there is limited information concerning tick-borne pathogens, Ehrlichia and Anaplasma spp., as well as genetic characterization of these species. We performed nested PCR for the gltA gene of the genus Ehrlichia spp. and the 16S rRNA gene of the genus Anaplasma spp. with blood samples from 400 apparently healthy dogs in Nay Pyi Taw area. These amplicon sequences were compared with other sequences from GenBank. Among the 400 blood samples from dogs, $3(0.75 \%)$ were positive for $E$. canis and $1(0.25 \%)$ was positive for A. platys. The partial sequences of the E. canis gltA and A. platys 16 SrRNA genes obtained were highly similar to E. canis and A. platys isolated from different other countries.
\end{abstract}

\section{Introduction}

Tick-borne bacteria and parasites are important pathogens of domestic dogs and are potentially of public health significance. At least five bacterial species, Ehrlichia canis, E. chaffeensis, E. ewingii, Anaplasma platys, and A. phagocytophilum, have been reported in domestic dogs [1]. E. canis is transstadially transmitted by the brown dog tick, Rhipicephalus sanguineus, and all feeding stages of tick can transmit the infection to susceptible dogs, and nymphal and adults can transmit E. canis for at least 155 days after detachment from an infected host [2]. Ehrlichia canis was the first Rickettsiales described in dogs and is the causal agent of canine monocytic ehrlichiosis (CME), which has a worldwide distribution, particularly in tropical and subtropical regions [3-5]. These bacteria are classified in the family Anaplasmataceae, which includes obligate intracellular prokaryotic parasites that reside within a parasitophorous vacuole [6]. In canine hosts, E. canis is infective for monocytes [7].

Anaplasma platys infections in dogs are distributed throughout the world. A. platys is the causative agent of canine infectious cyclic thrombocytopenia, which infects the platelets, but infected dogs showed no clinical signs [8]. A. platys infection is difficult to detect not only "in vivo" because of the low bacteremias but also serologically because of cross-reaction with other Anaplasma species $[9,10]$. Thus, a PCR assay is a reliable method for the detection of $A$. platys infection in dogs [11].

The objectives of this study were to determine the presence of E. canis and A. platys in dogs and to compare Myanmar isolates with those from other regions. Herein, we used nested PCR and phylogenetic analysis to detect the molecular characteristics of E. canis and A. platys infections from dogs in the Nay Pyi Taw area, Myanmar. 


\section{Materials and Methods}

2.1. Study Site and Sample Collection. This study was conducted in four townships: Lewe $\left(19.6349^{\circ} \mathrm{N}, 96.1076^{\circ} \mathrm{E}\right)$, Pyinmana $\left(19.7414^{\circ} \mathrm{N}, \quad 96.2004^{\circ} \mathrm{E}\right)$, Tatkon $\left(20.1284^{\circ} \mathrm{N}\right.$, $\left.96.1527^{\circ} \mathrm{E}\right)$, and Zay Yar Thi Ri (19.62 $\left.\mathrm{N}, 96.02^{\circ} \mathrm{E}\right)$ (Figure 1). Between December 2016 and March 2017, blood samples were collected from 400 apparently healthy dogs. From the urban and rural areas of each township, 100 dogs were sampled. Most of the dogs are free roaming in rural Myanmar, while they belong to someone. Before taking blood samples, we explained our aim of the study to the owner, and we have already obtained consent for the experiment from the dog owners. Blood collection (approximately $3 \mathrm{ml}$ ) was performed from the sphenoid vein and jugular vein and put into ethylene diamine tetraacetic acid (EDTA) tubes. All collected samples were transferred to the laboratory at $4^{\circ} \mathrm{C}$. Within $24 \mathrm{hr}$ of sample collection, DNA extraction was conducted. During blood collection, dogs were examined for the presence of ticks, and if present, ticks were collected in plastic containers containing a small piece of wet sponge for further taxonomic identification.

2.2. DNA Extraction from Canine Blood. Extraction of DNA from the blood samples was conducted by using a commercial DNA extraction reagent (DNAzol ${ }^{\circledR}$ ) (Molecular Research Center, Inc., USA) according to the manufacturer's instructions [12]. The volume of blood used for DNA extraction was $100 \mu \mathrm{l}$. The extracted DNAs were eluted in $200 \mu \mathrm{l}$ elution buffer and stored at $-80^{\circ} \mathrm{C}$. DNA concentration was estimated using a NanoDrop 2000 spectrophotometer (ThermoFisher Scientific, MA, USA).

\subsection{Polymerase Chain Reaction (PCR) to Amplify Ehrlichia} and Anaplasma spp. For Ehrlichia spp., seminested PCR amplification of the gltA gene fragment was performed by using a SimpliAmp Thermal cycler (Applied Biosystem, USA) as previously described [13]. Outer primers, EHRCS$131 \mathrm{~F}$ (CAGGATTTATGTCTACTGCTGCTTG) and EHRCS-1226R (CCAGTATATAAYTGACGWGGACG), were used for the amplification of the first-round product (1,096bp), and inner primers, EHRCS-131F (CAGGATTTATGTCTACTGCTGCT TG) and EHRCS-879R (TIGCKCCACCATGAGCTG), were used for the amplification of the second-round product (748bp). For Anaplasma spp., seminested PCR amplification of the 16S rRNA gene fragment was performed according to Inokuma et al. [14]. Outer primers, fD1 (AGAGTTTGATCCTGGCTC AG) and EHR16SR (TAGCACTCATCGTTTA CAGC), were used for the first-round product $(1,000 \mathrm{bp})$, and inner primers, EHR16SD (GGTACC(C/T)ACAGAAGAAGTCC) and Rp2 (ACGGCTACCTTGTTACGACTT), were used for the second-round product $(1,000 \mathrm{bp})$. PCR mixture contained approximately $20-100 \mathrm{ng}$ of extracted DNA, $0.3 \mu \mathrm{M}$ of each primer, $0.025 \mathrm{U} / \mu \mathrm{L}$ of Tks Gflex ${ }^{\mathrm{TM}}$ DNA polymerase (Takara Bio Inc., Tokyo, Japan), and $1 \times$ Gflex buffer in a volume of $25 \mu \mathrm{L}$. For both species, cycling conditions were denaturation for $1 \mathrm{~min}$ at $94^{\circ} \mathrm{C}$, followed by $98^{\circ} \mathrm{C}$ for $10 \mathrm{~s}$. The annealing temperature used was $50^{\circ} \mathrm{C}$ for $15 \mathrm{~s}$ for Ehrlichia spp. and $55^{\circ} \mathrm{C}$ for Anaplasma spp., followed by $68^{\circ} \mathrm{C}$ for $90 \mathrm{~s}$ for 40 cycles, and a final extension for $5 \mathrm{~min}$ at $68^{\circ} \mathrm{C}$. The PCR products were visualized by electrophoresis on $1.5 \%$ agarose gels stained with RedSafe (NIPPON Genetics, Duren, Germany).

2.4. Sequencing and Phylogenetic Analysis. Positive PCR products were purified using the NucleoSpin ${ }^{\circledR}$ and PCR Clean-up Kit (MACHEREY-NAGEL, Duren, Germany) according to the manufacturer's instructions. Purified PCR products were sequenced with the ABI 3130 genetic analyzer (Model 3130; Applied Biosystems, Foster City, CA, USA) with forward and reverse primers. Nucleotide sequences were compared with GenBank entries using NCBI BLAST (http://www.ncbi.nlm.nih.gov/blast). Phylogenetic analysis is performed using the DNA sequence.

Multiple sequence alignments of positive amplicons and gltA and 16S rRNA sequences from GenBank were performed using the ClustalW Version 1.8 [15]. Phylogenetic trees were inferred using neighbor-joining (NJ) analysis using MEGA software version 7.0 [16]. The distance matrix of nucleotide divergences was calculated according to Kimura's two-parameter model furnished by MEGA. A bootstrap resampling technique of 1000 replications was performed to statistically support the reliabilities of the nodes on the trees.

\section{Ethical Considerations}

Experiments were carried out in accordance with the guidelines laid down by the Institutional Ethics Committee. All studies using animal subjects were approved by the Ethics Committee of University of Veterinary Science, Nay Pyi Taw, Myanmar (approval no. 309/Katha (postgraduate)/ 2016.

\section{Results and Discussion}

Of the 400 dogs analyzed, 3 samples $(0.75 \%)$ were positive for E. canis, while 1 sample (0.25\%) was positive for A. platys (Table 1, Figures 2(a) and 2(b)). Descriptive data of sampled dogs and tick infestation are shown in Table 2. All PCRpositive samples for A. platys and E. canis were confirmed by sequencing results.

In the phylogenetic trees based on gltA genes, E. canis was detected in dogs T1, T8, and T9 grouped in the same cluster as other E. canis strains, supported with a $100 \%$ bootstrap value. The $A$. platys 16 SrRNA gene from dog Z4 was found in the same cluster as other A. platys strains, supported with a $100 \%$ bootstrap value. The sequences obtained were similar to those of E. canis strains from Philippines, Italy, Spain, France, China, and Thailand (GenBank accession no. JN391409, AY647155, AY615901, AF304143, KX987357, KU765198, and KU765199) with similarities of $98.46-100 \%$ (Figure 3 ). A. platys $16 \mathrm{~S}$ rRNA sequences obtained were similar to those of $A$. platys strains from India, Thailand, Italy, Okinawa, Croatia, China, Spain, and South Africa (GenBank accession no. KT982643, 


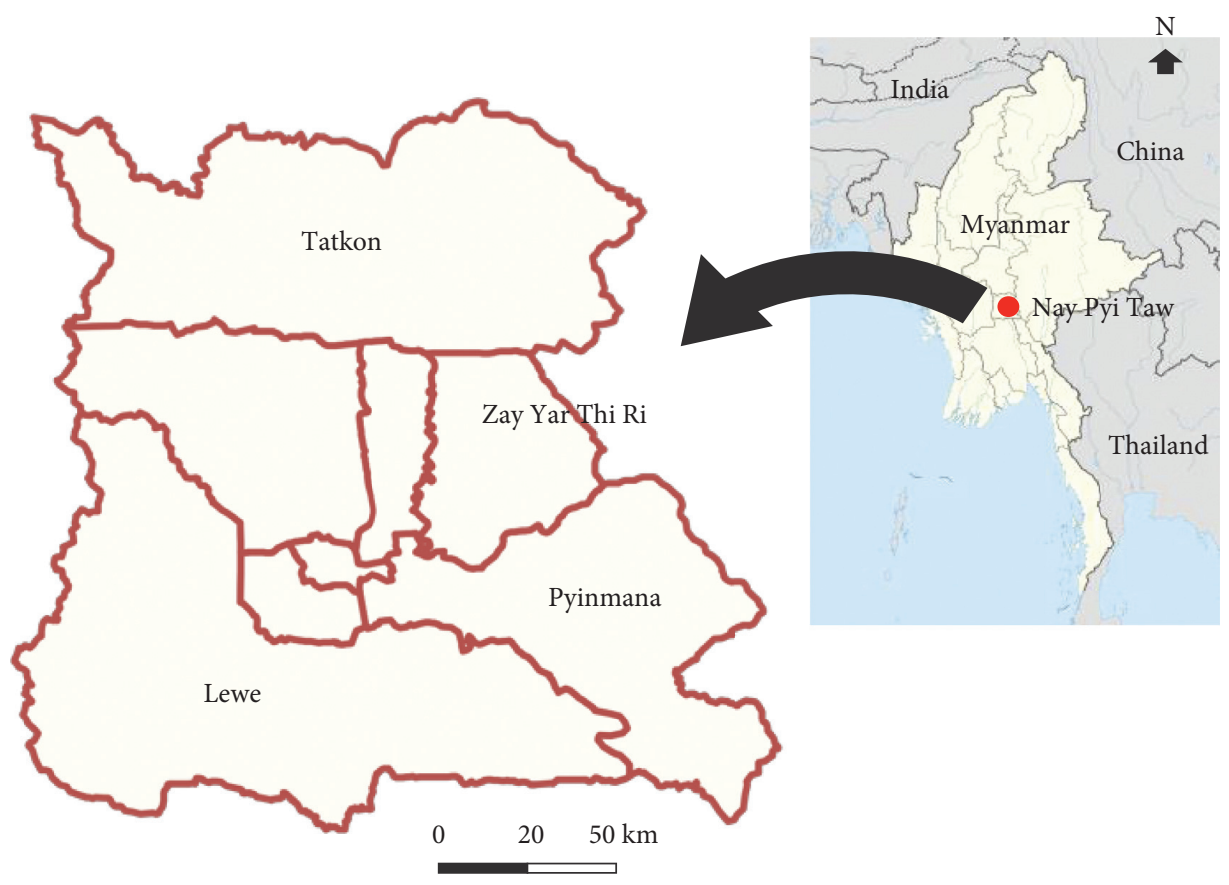

Figure 1: Map of the sampling area.

Table 1: Prevalence of E. canis and A. platys in four townships within Nay Pyi Taw area.

\begin{tabular}{lccc}
\hline Location & No. of collected samples & \multicolumn{2}{c}{ No. (\%) of positive samples } \\
& & E. canis & $0(0)$ \\
Pyinmana & 100 & $0(0)$ & $1(1)$ \\
Zay Yar Thi Ri & 100 & $0(0)$ & $0(0)$ \\
Lewe & 100 & $0(0)$ & $0(0)$ \\
Tatkon & 100 & $3(3)$ & $1(0.25)$ \\
Total & 400 & $3(0.75)$ & A.tatys \\
\hline
\end{tabular}

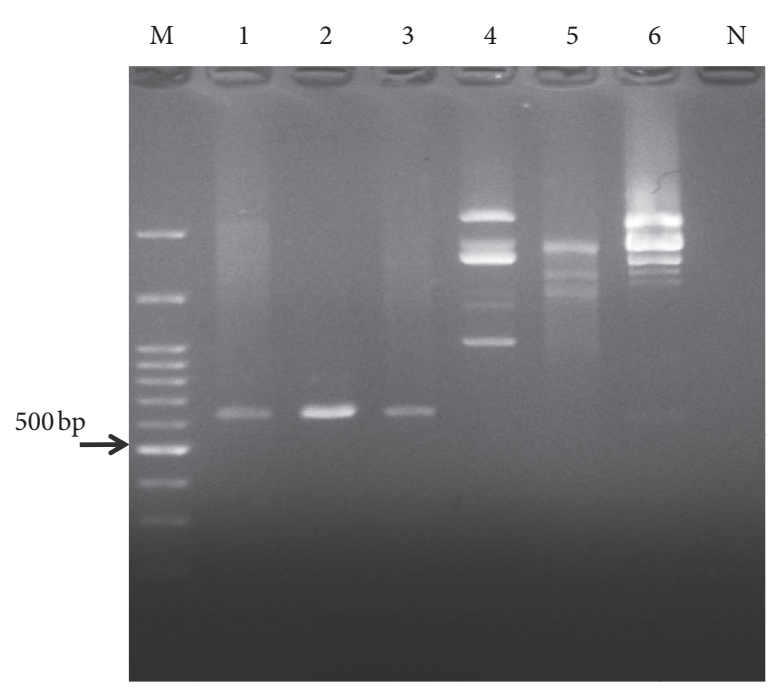

(a)

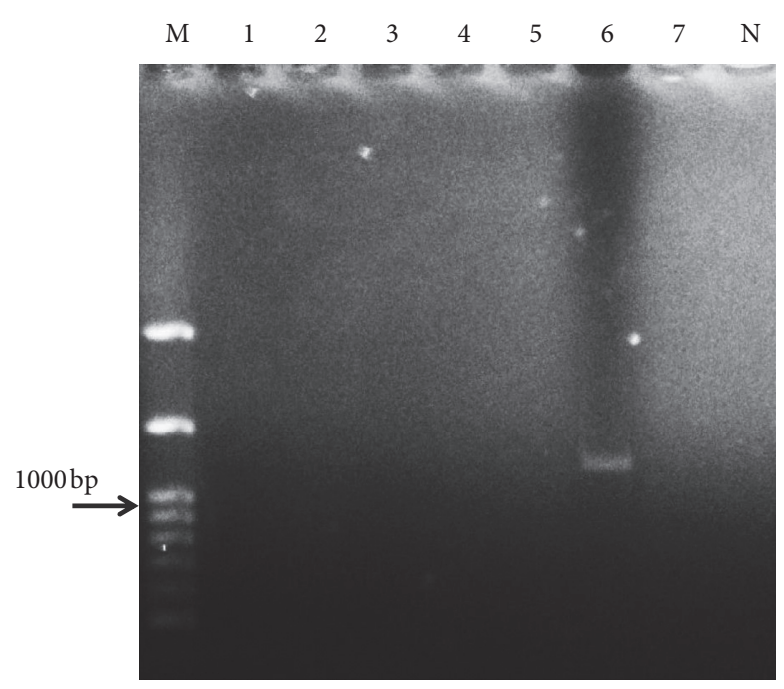

(b)

FIgURE 2: Gel electrophoresis results for nested PCR of E. canis (a): lanes 1-3=positive samples, lanes 4-6=negative samples, and $N=$ negative control, and $A$. platys (b): lane $6=$ positive, lanes $1-5$, and $7=$ negative samples, and $N=$ negative control; $M=100 \mathrm{bp}$ marker. 
TABLE 2: Description of sampled dogs and tick infestation.

\begin{tabular}{lccccccrrr}
\hline \multirow{2}{*}{ No. of examined dogs } & \multicolumn{2}{c}{ Age } & \multicolumn{2}{c}{ Sex } & \multicolumn{3}{c}{ Breed } & \multicolumn{2}{c}{ Tick infestation } \\
& $>1$ year & $<1$ year & Male & Female & Local & Exotic & Yes & No \\
\hline 400 & 251 & 149 & 220 & 180 & 400 & 0 & 44 & 356 \\
\hline
\end{tabular}

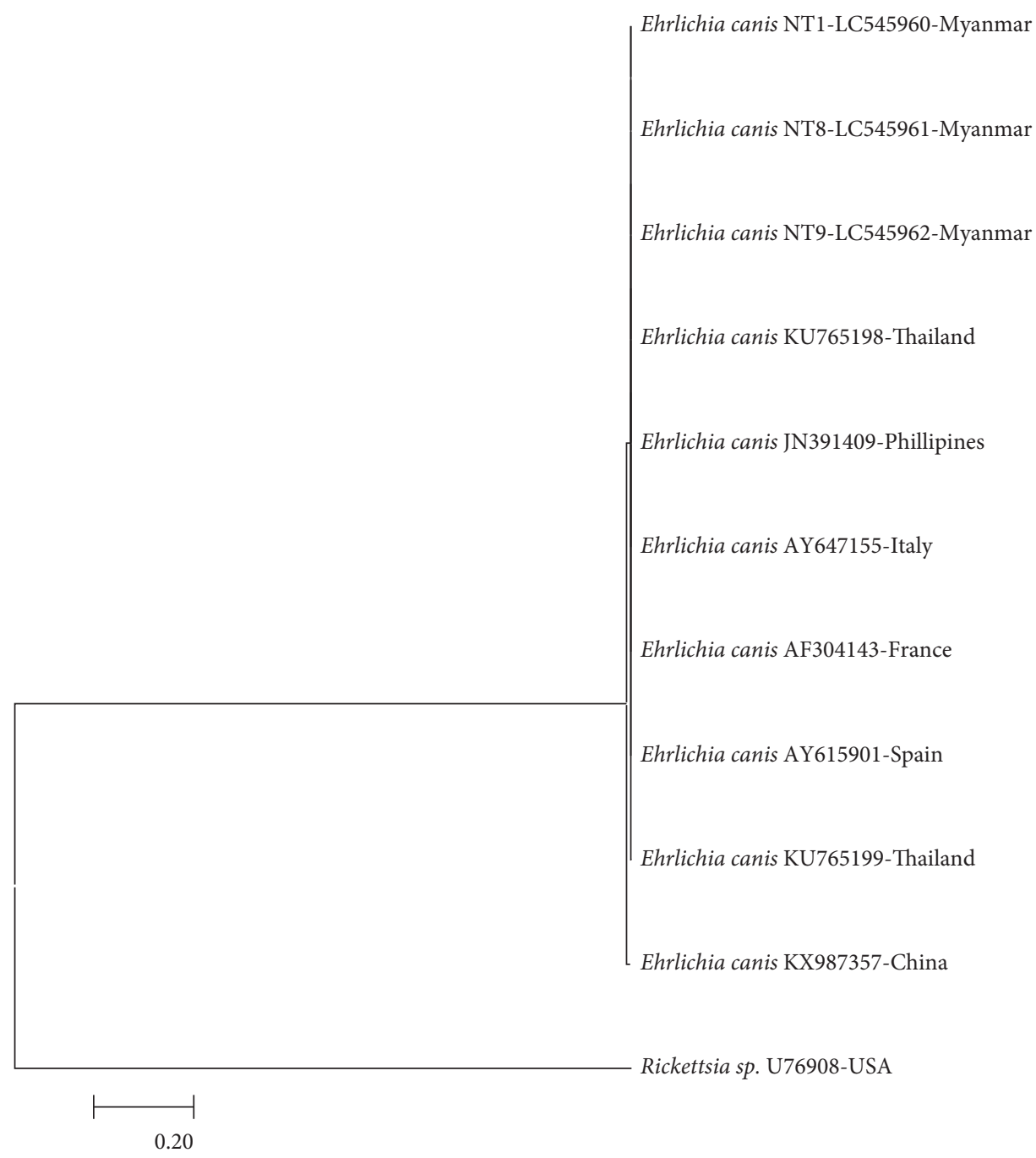

FIGURE 3: Phylogenetic tree based on the Ehrlichia gltA sequence. Sequences from the Ehrlichia genera were compared with the neighborjoining method with distance matrix calculation by Kumar-two parameters, operated by MEGA software (Version 7), using Rickettsia sp. as the outgroup. Scale bar indicates the number of mutations per sequence position. The numbers at the nodes represent the percentage of 1000 bootstrap resamplings.

EF139459, EU439943, AF536828, KY114935, KJ659044, KX987336, AY530806, and KC189853) with similarities of 99.68-100\% (Figure 4). The results from phylogenetic analysis confirmed that the amplified genes belong to the respective species. Sequences generated in the present study have been submitted to GenBank under accession numbers LC545959 to LC545962.

In this study, molecular identification from 400 local dog samples demonstrated a prevalence of $0.75 \%$ for E. canis infection and $0.25 \%$ for A. platys infection. There was no mixed infection in this study. According to the findings of this study, E. canis was found as more common canine tick-borne pathogen when compared to Anaplasma spp. In this study, the present results indicate a low prevalence of subclinical infection in dogs. In Turkey, the prevalence of E. canis from asymptomatic dogs was $4.9 \%$, A. platys was $0.5 \%$, and mixed infections of E. canis and A. platys were detected as $0.3 \%$ [17]. In Brazil, only $4.8 \%$ of the dogs were seroreactive to E. canis [18]. Previous studies have described that the molecular prevalence of E. canis ranged from $3.1 \%$ to $88 \%$ [19-23]. The variation might be due to the sample size, climatic conditions that directly influence the tick population, and the time of sample collection. 


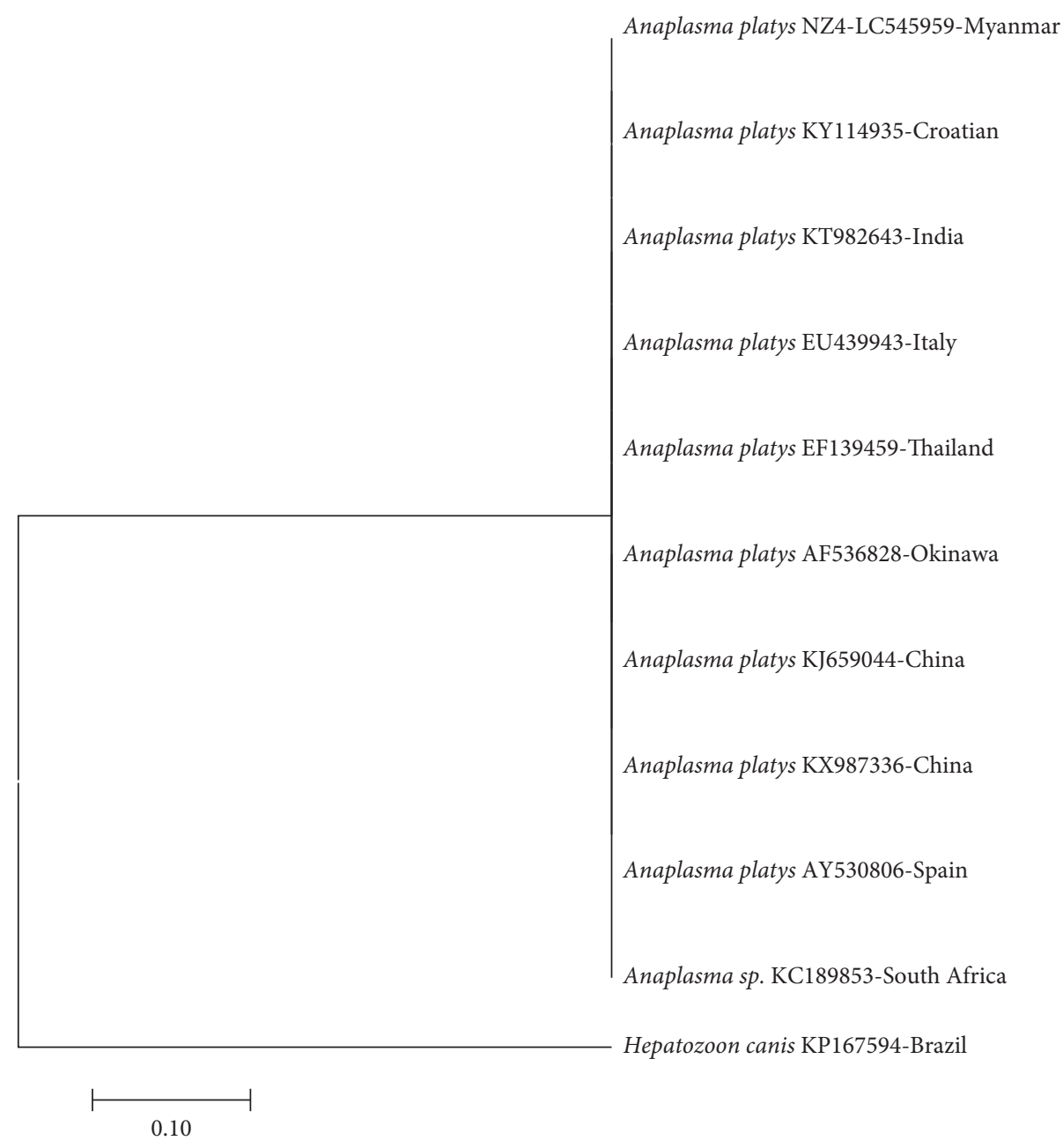

FIgURe 4: Phylogenetic tree based on the Anaplasma 16S rRNA sequence. Sequences from the Anaplasma genera were compared with the neighbor-joining method with distance matrix calculation by Kumar-two parameters, operated by MEGA software (version 7), using Hepatozoon canis as the outgroup. Scale bar indicates the number of mutations per sequence position. The numbers at the nodes represent the percentage of 1000 bootstrap resamplings.

A higher prevalence of E. canis and A. platys was also reported by some workers. In Praia, Austria, Gotsch et al. [24] indicated that the PCR examination for E. canis in dogs was $26.2 \%$ and A. platys was $7.7 \%$. In North Carolina, USA, $33 \%$ of 27 dogs was A. platys PCR-positive [25]. In Okinawa, Japan, 32\% of 200 stray dogs was positive by A. platysspecific PCR [26]. In fact, in the previous studies, the positive dogs were sick animals with clinical signs compatible with vector-borne diseases and admitted for medical treatment, while in the present study, all the dogs sampled were apparently healthy.

Anaplasma platys is a thrombocytotrophic bacteria of dogs that is characterized by clinical abnormalities such as fever, anorexia, petechial haemorrhages, and uveitis [27]. The detection of A. platys infection in dogs was the first time in Myanmar. In this study, it was lower prevalence of A. platys $(0.25 \%)$ than that in Italy (4\%), Nigeria (6.6\%), and Venezuela (16\%). In Portugal, A. platys DNA has been detected in clinically suspected dogs living in the north and south of Portugal [28], while the overall national seroprevalence of Anaplasma spp. has ranged from $4.5 \%$ in apparently healthy to $9.2 \%$ in clinically suspect dogs [29] The lower prevalence of $A$. platys in this study might be due to different DNA extraction methods, and the local breed of the examined dogs in this study seemed to be genetically resistant to tick-borne pathogens. In this study, the older dogs were more likely to be positive and could have a greater risk of tick-borne diseases. Moreover, younger dogs might be maternally immune to tick infection. Since local dogs are free roaming in rural areas, they have never been treated or removed of ticks, and they may naturally be resistant to tickborne diseases. However, further studies are necessary to identify the infections of E. canis and A. platys from both ticks and hosts.

In this study, all the tick samples collected during sampling were morphologically and molecularly diagnosed R. sanguineus (data not shown). However, the occurrence of tick infestation in dogs in the study area was low $(11 \%, 44 /$ 400) [30]. A total of 237 ticks were collected from 44 dogs with an average of 4-5 ticks per dog. Three out of four 
positive dogs were infected with ticks in the studied areas. These data suggest that E. canis and A. platys might be shared by the same vector, $R$. sanguineus. In Myanmar, Chel [31] studied that the prevalence of $R$. sanguineus tick in Nay Pyi Taw area was $0 \%$ in the summer season, $84.7 \%$ in the rainy season, and $15.3 \%$ in the winter season. Asebe et al. [32] also discussed that in tropical climates, there is a marked decrease in tick population at the end of the rainy season and with progressive fall to almost zero in the dry season. In fact, as stated by Huang et al. [33], one of the reasons for the low prevalence of $E$. canis and A. platys might also be due to a very small number of $R$. sanguineus ticks collected in the present study. Moreover, this might be due to climatic conditions during the sampling period (from December to March), which were not favourable for development and survival of $R$. sanguineus.

The partial sequences of the gltA and 16SrRNA genes obtained in this study were highly similar to strains of E. canis and A. platys isolated from different other countries. This implied that the E. canis and A. platys isolates found in Myanmar were not divergent from the strains of other countries. This might be due to the fact that transboundary movement of domestic and wild animals might carry infected ticks between Myanmar and neighboring countries. The vectors might distribute genetically similar pathogens among these countries.

\section{Conclusion}

The findings of this study are basic information regarding E. canis and A. platys infection in Myanmar. Moreover, further research related to the genetic diversity of $E$. canis and A. platys from another area of Myanmar should be conducted.

\section{Data Availability}

Sequences generated in the present study have been submitted to GenBank under accession numbers LC545959 to LC545962.

\section{Conflicts of Interest}

The authors declare that they have no conflicts of interest.

\section{Authors' Contributions}

All authors contributed equally to the research and writing of the manuscript.

\section{Acknowledgments}

This research was supported in part by the Laboratory of Parasitology, Graduate School of Veterinary Medicine, Hokkaido University, Japan. The authors are grateful to Prof. Dr. Ken Katakura and Associate Prof. Dr. Ryo Nakao, Laboratory of Parasitology, Graduate School of Veterinary Medicine, Hokkaido University, Japan, for their partial support and cooperation.

\section{References}

[1] M. Perez, M. Bodor, C. Zhang, Q. Xiong, and Y. Rikihisa, "Human infection with Ehrlichia canis accompanied by clinical signs in Venezuela," Annals of the New York Academy of Sciences, vol. 1078, no. 1, pp. 110-117, 2006.

[2] W. L. Nicholson, K. E. Allen, J. H. McQuiston, E. B. Breitschwerdt, and S. E. Little, "The increasing recognition of rickettsial pathogens in dogs and people," Trends in Parasitology, vol. 26, no. 4, pp. 205-212, 2010.

[3] C. N. Gutiérrez, M. Martínez, E. Sánchez et al., "Cultivation and molecular identification of Ehrlichia canisand Ehrlichia chaffeensisfrom a naturally co-infected dog in Venezuela," Veterinary Clinical Pathology, vol. 37, no. 3, pp. 258-265, 2008.

[4] R. F. Silva-Molano, N. Sánchez-Ucrós, and A. M. LoaizaEcheverri, "Remove from marked records recurrence report of Ehrlichia canis in dog blood samples in Cali, Colombia," Veterinaria Y Zootecnia, vol. 2, no. 1, pp. 27-31, 2010.

[5] P. J. Kelly, C. Xu, H. Lucas, and A. Loftis, "Ehrlichiosis, babesiosis, anaplasmosis and hepatozoonosis in dogs from st. Kitts, west indies," PLoS One, vol. 8, no. 1, Article ID e53450, 2013.

[6] J. S. Dumler, A. F. Barbet, C. P. Bekker et al., "Reorganization of genera in the families Rickettsiaceae and Anaplasmataceae in the order Rickettsiales: unification of some species of Ehrlichia with Anaplasma, Cowdria with Ehrlichia and Ehrlichia with Neorickettsia, descriptions of six new species combinations and designation of Ehrlichia equi and "HGE agent"as subjective synonyms of Ehrlichia phagocytophila," International Journal of Systematic and Evolutionary Microbiology, vol. 51, no. 6, pp. 2145-2165, 2001.

[7] E. Greene and J. W. Harvey, "Canine Ehrlichiosis," in Clinical Microbiology and Infectious Diseases of the Dog and Cat, C. E. Greene, Ed., pp. 137-148, W. B. Saunders, Philadelphia, PA, USA, 1990.

[8] J. W. Harvey, C. F. Simpson, and J. M. Gaskin, "Cyclic thrombocytopenia induced by a Rickettsia-like agent in dogs," Journal of Infectious Diseases, vol. 137, no. 2, pp. 182-188, 1978.

[9] S. Harrus, T. Waner, and H. Bark, "Canine monocytic ehrlichiosis update," Compendium for Continuing Education for the Practicing Veterinarians, vol. 19, pp. 431-444, 1997.

[10] J. P. Beaufils, H. Inokuma, and J. Martin-Granel, “Anaplasma platys (Ehrlichia platys) infection in a dog in France: description of the case and characterization of the agent," Revue de médecine vétérinaire, vol. 153, pp. 85-90, 2002.

[11] A. R. Martin, G. K. Brown, R. Hugh Dunstan, and T. K. Roberts, "Anaplasma platys: an improved PCR for its detection in dogs," Experimental Parasitology, vol. 109, no. 3, pp. 176-180, 2005.

[12] R. Nakao, E. Y. Stromdahl, J. W. Magona et al., "Development of loop-mediated isothermal amplification (LAMP) assays for rapid detection of Ehrlichia ruminantium," BMC Microbiology, vol. 10, no. 1, p. 296, 2010.

[13] A. D. Loftis, W. K. Reeves, J. P. Spurlock et al., "Infection of a goat with a tick-transmitted Ehrlichia from Georgia, USA., that is closely related to Ehrlichia ruminantium," Journal of Vector Ecology, vol. 31, no. 2, pp. 213-223, 2006.

[14] H. Inokuma, K. Ohno, T. Onishi, D. Raoult, and P. Brouqui, "Detection of ehrlichial infection by PCR in dogs from Yamaguchi and Okinawa Prefecture," The Journal of Veterinary Medical Science, vol. 63, no. 7, pp. 815-817, 2001.

[15] J. D. Thompson, D. G. Higgins, and T. J. Gibson, "Clustal W: improving the sensitivity of progressive multiple sequence 
alignment through sequence weighting, position-specific gap penalties and weight matrix choice," Nucleic Acids Research, vol. 22, no. 22, pp. 4673-4680, 1994.

[16] S. Kumar, G. Stecher, and K. Tamura, "MEGA7: molecular evolutionary genetics analysis Version 7.0 for bigger datasets," Molecular Biology and Evolution, vol. 33, no. 7, pp. 1870-1874, 2016.

[17] M. Aktas, S. Özübek, K. Altay et al., "Molecular detection of tick-borne rickettsial and protozoan pathogens in domestic dogs from Turkey," Parasites \& Vectors, vol. 8, no. 1, p. 157, 2015.

[18] T. B. Saito, C. E. Larsson, M. B. Labruna et al., "Canine infection by Rickettsiae and Ehrlichiae in Southern Brazil," The American Journal of Tropical Medicine and Hygiene, vol. 79, no. 1, pp. 102-108, 2008.

[19] G. L. Murphy, S. A. Ewing, L. C. Whitworth, J. C. Fox, and A. A. Kocan, "A molecular and serologic survey of Ehrlichia canis, E. chaffeensis, and E. ewingii in dogs and ticks from Oklahoma," Veterinary Parasitology, vol. 79, no. 4, pp. 325-339, 1998.

[20] A. S. Dagnone, H. S. A. de Morais, M. C. Vidotto, F. S. Jojima, and O. Vidotto, "Ehrlichiosis in anemic, thrombocytopenic, or tick-infested dogs from a hospital population in South Brazil," Veterinary Parasitology, vol. 117, no. 4, pp. 285-290, 2003.

[21] P. P. V. P. Diniz, D. S. Schwartz, H. A. S. Morais, and E. B. Breitschwerdt, "Surveillance for zoonotic vector-borne infections using sick dogs from southeastern Brazil," Vector Borne and Zoonotic Diseases, vol. 7, no. 4, pp. 689-697, 2007.

[22] N. Alexandre, A. S. Santos, M. S. Núncio, R. d. Sousa, F. Boinas, and F. Bacellar, "Detection of Ehrlichia canis by polymerase chain reaction in dogs from Portugal," The Veterinary Journal, vol. 181, no. 3, pp. 343-344, 2009.

[23] G. C. F. d. Silva, A. d. N. Benitez, A. Girotto et al., "Occurrence of Ehrlichia canis and Anaplasma platys in household dogs from northern Parana," Revista Brasileira de Parasitologia Veterinária, vol. 21, no. 4, pp. 379-385, 2012.

[24] S. Götsch, M. Leschnik, G. Duscher, J. P. Burgstaller, W. Wille-Piazzai, and A. Joachim, "Ticks and haemoparasites of dogs from Praia, Cape Verde," Veterinary Parasitology, vol. 166, no. 1-2, pp. 171-174, 2009.

[25] S. K. Kordick, E. B. Breitschwerdt, B. C. Hegarty et al., "Coinfection with multiple tick-borne pathogens in a Walker Hound kennel in North Carolina," Journal of Clinical Microbiology, vol. 37, no. 8, pp. 2631-2638, 1999.

[26] Y. Motoi, H. Satoh, H. Inokuma et al., "First Detection of Ehrlichia platysin Dogs and Ticks in Okinawa, Japan," Microbiology and Immunology, vol. 45, no. 1, pp. 89-91, 2001.

[27] J. Kamani, G. Baneth, and K. Y. Mumcuoglu, "Molecular detection and characterization of tick-borne pathogens in dogs and ticks from Nigeria," PLoS Neglected Tropical Diseases, vol. 7, no. 3, Article ID e2108, 2013.

[28] Y. Yisaschar-Mekuzas, C. L. Jaffe, J. Pastor, L. Cardoso, and G. Baneth, "Identification of Babesia species infecting dogs using reverse line blot hybridization for six canine piroplasms, and evaluation of co-infection by other vector-borne pathogens," Veterinary Parasitology, vol. 191, no. 3-4, pp. 367-373, 2013.

[29] A. S. Santos, N. Alexandre, R. Sousa, M. S. Nuncio, F. Bacellar, and J. S. Dumler, "Serological and molecular survey of Anaplasma species infection in dogs with suspected tickborne disease in Portugal," Veterinary Record, vol. 164, no. 6, pp. 168-171, 2009.
[30] M. M. Hmoon, L. L. Htun, S. S. Wai, and M. J. Thu, "Morphological and molecular identification of ticks infested in stray dogs within Nay Pyi Taw area, Myanmar," South Asian Journal of Life Sciences, vol. 6, no. 2, pp. 41-45, 2018.

[31] H. M. Chel, "Study on morphological identification of ticks associated with seasonal occurrence within Nay Pyi Taw area," MSc Thesis, University of Veterinary Science, Nay Pyi Taw, Myanmar, 2015.

[32] G. Asebe, Y. Hailu, A. Basu, and D. Shibru, "Overview of the biology, epidemiology and control methods against hard ticks: A review," Global Journal of Science Frontier Research, vol. 16, pp. 2249-4626, 2016.

[33] H. Huang, A. Unver, M. J. Perez, N. G. Orellana, and Y. Rikihisa, "Prevalence and molecular analysis of Anaplasma platys in dogs in Lara, Venezuela," Brazilian Journal of Microbiology, vol. 36, no. 3, pp. 211-216, 2005. 